\title{
Design and Development of Techniques for Equipment Health Monitoring System
}

\author{
Vasireddy Prabha Kiranmai, Sharmitha S Bysani, Vijaya Kumar B P, Kusuma S M
}

\begin{abstract}
Machines in Industries are often subjected to enormous wear and tear, which if unnoticed, may lead to production delays and increased maintenance cost. Machines must be able to analyse and provide statistics about its health, so that preventive measures can be taken to avoid catastrophes in the industries. Thus, there is a need of automated fault detection and prediction of system's condition. The concept of equipment health monitoring is a crucial step in the field of research and development in the manufacturing industries. This equipment makes it handy in situations where machines require continuous monitoring and is difficult for humans to provide such attention , especially in the case of unmanned vehicles.
\end{abstract}

Prediction of the status of equipment by acquisition of data from industrial machinery is the critical step in building such a system. Health of machines can be estimated by the data collected by the sensors-temperature, accelerometer, etc integrated with an embedded computing system, like a Raspberry Pi. This IoT model consisting of embedded system with wireless connectivity collects real time data from the equipment/machinery used in industries. This data is used to analyse and predict the health of the equipment, examine the steady-state characteristics using Machine Learning technique, Hidden Markovian Model.

The concept of the proposed IoT model is evaluated over a conveyor belt test rig under various conditions, like different loads placed on various locations of conveyor belt and the belt is made to run at different speeds and data is collected over all these conditions. Then, a data model is created using Hidden Markovian Model which is further used in predicting the state of the belt based on the sequential data, here it is the sensor data. Given a state of the belt, this model can predict whether the belt is in proper condition or not, and if human intervention is required. Thus, at any point of time, having this setup on the machinery which needs to be monitored can be used in predicting the faults and notifying the user in case of any faulty behaviour or malfunctioning of machines. This setup can be used for any machines which are subjected to any motion, vibration and thermal changes. This helps in creating a completely automated fault detection systems in the present Industries.

Keywords: accelerometer, automated fault detection, condition of machine, equipment health monitoring, IoT, Hidden Markovian Model, sensor

Revised Manuscript Received on December 22, 2019

* Correspondence Author

Vasireddy Prabha Kiranmai, Information Science and Engineering, Ramaiah Institute of Technology, Bangalore, India. Email: kiranmaivrpk@gmail.com

Sharmitha S Bysani, Information Science and Engineering, Ramaiah Institute of Technology, Bangalore, India. Email: sharmitha25@gmail.com

Vijaya Kumar B P, Information Science and Engineering, Ramaiah Institute of Technology, Bangalore, India. Email: vijay.beekan@gmail.com

Kusuma S M, Telecommunication Engineering, Ramaiah Institute of Technology, Bangalore, India. Email: kusum.sugur@yahoo.in

\section{INTRODUCTION}

$\mathrm{M}$ achines are in motion, either vibrational or rotational. They undergo wear and tear, and often deteriorate with time and usage. These failures are very frequent and may go unnoticed, leading to the breakdown of the entire machine. Identification of this faults is critical, as worn out equipment consume more energy than the properly functioning machines. This would affect the quality, cost and productivity. Due to the large number of equipments present in Industries, monitoring the condition of every machine manually is tedious. Instead, a setup which could monitor the health of the machine on continuous intervals and only notify when fault has occurred and human intervention is required, would ease our task.

Machines must be able to analyse and provide statistics about its health, so that preventive measures can be taken, this could save us from the damage of equipment, well in advance. This idea comes into great favour in case of unmanned vehicles where there is no human intervention to monitor the damage. Monitoring the condition of machines along with managing the labour work requires additional manpower, which is quite expensive. In addition, the strategy to increase the lifetime of an equipment is to run regular checks to ensure that the equipment is in good condition. This can be done by the use of intelligent predictive technologies and tools. Deployment of these tools and techniques can detect malfunctions or potential breakdowns in machines.

The motivation of this project is to eliminate the manual intervention in check-up of the health of machines in Industries. Oil refineries have conveyor belts stretching over kilometres, and it is practically impossible for humans to examine these belts without any error and with accuracy. Also, this project aims to predict if the state of the machine on which it is operating is ideal with respect to the conditions of the surrounding in which it is operated. This would give the user an idea on when the machine would breakdown. Therefore, it aims to analyse and provide statistics about the equipment's health.

The objective of this project is to record the vibrations and temperature of the machine (belt) and retrieved through a data cloud which can then be converted to the desired format and be provided as input to the algorithm and then study the different equipment health monitoring methodologies and techniques to the acquired data from machineries like 


\section{Design and Development of Techniques for Equipment Health Monitoring System}

Conveyor Belt using sensors and implement the proposed equipment Health Monitoring System using IoT and Machine Learning technique, Hidden Markovian Prediction Model to learn the data model and further predict the state of the system, from the known data model.

The paper is organized as follows: Firstly, we discuss few related works in section II, Secondly, we have section III where the system model is explained. Thirdly, we have Section IV which deals with the implementation of the idea and then, section $\mathrm{V}$ is regarding the experimental results we have obtained when this above explained proof of concept is implemented in the laboratory. Finally, the Future Work and Conclusions section is discussed with the finding of this project, are explained in section VI.

\section{RELATED WORK}

IoT and wireless sensor networks act as base for equipment health monitoring. Here, we provide a brief insight of the papers we have referred and taken some inspiration for this project.

Firstly, the case study that was referred is, In [1], the sensors are positioned near the source of vibrations to monitor the vibrations of the bridge continuously. To identify the condition of the bridge, the signals obtained are processed to extract the features of the bridge. The displacement and acceleration of bridge responses are measured by the sensors. This data is sent to the local server where the data was interpreted using neural network techniques. The aim is to determine how the acceleration and displacement data domain would perform, when given as input to the applied neural networks. The architecture of the neural network model consists of an input layer, one or two hidden layers with $\mathrm{n}$ neurons and an output layer. The time- acceleration and the time- displacement domains of the bridge due to earthquake loads form the input layer, while the bridge condition level determined using finite-element analysis software constitutes the output layer. The bridge condition is classified in a range 0 to 3 , which state the extent of bridge health condition starting from safe to high risk level. Thus, neural network helps in identifying the condition of a bridge at particular time due to an earthquake, and thereby the repair or maintenance of the bridges can be performed to avoid any damages to the bridge

Secondly, another case study which was referred is the Structural health monitoring via micro electromechanical system accelerometers was carried out in [2] which gives a brief description of how these devices can be embedded within wireless sensor networks in order to allow the captured data measurements to be transmitted wirelessly to the local server. WSN technology was used for structural health monitoring due to its ease of installation with minimal structural intervention and low cost. This paper gives a review on the recent developments in the area of Structural Health Monitoring using WSNs. This paper also demonstrates the use of the following sensors to capture various parameters related to a structure, like Fiber optic sensors (FOS), piezoelectric sensors and magneto resistive sensors which can be used to predict the health of the structures by sensing the chemical and physical parameters related to it.
Thirdly, Paper [3] uses the probabilistic robustness theory to design a system for fault detection and monitoring. The background related to this work is an $R \& D$ project whose objective meets the following requirements:

- Continuous monitoring of the equipment aims to identify the changes taking place in the conveyor belt.

- This system features in detecting faults in the conveyor belt while it is in operation.

Finally, In paper [4], the author has depicted a hardwaresoftware model which can be deployed remotely to monitor the machines and collect the data from it. The hardware components include a PCB with an IEEE 802.15.4compatible microcontroller, Micro-USB connector, battery, battery charger/monitor, humidity/temperature sensor, IMU, and a MEMS microphone. Wireless control of the sensors and data collection is supported by the software. The user interface of the entire system allows 3-dimensional view of the collected data and the entire setup of the system. In case of rotating machinery, two different motor setups are arranged to test this equipment in the laboratory to ensure the proper functioning of the experiment.

Based on the following literature survey in this domain of equipment health monitoring system, we have proposed a model which could meet the objective of the project along with the good accuracy and a setup which is feasible. We have conducted this proof of concept over an industrial machine, Conveyor belt spread about $10 \mathrm{~m}$ in length.

\section{SYSTEM MODEL}

\section{A. Proposed Model}

An intelligent system for equipment health monitoring to analyse and make optimal data predictions. The following is a diagrammatic representation of the proposed system model:

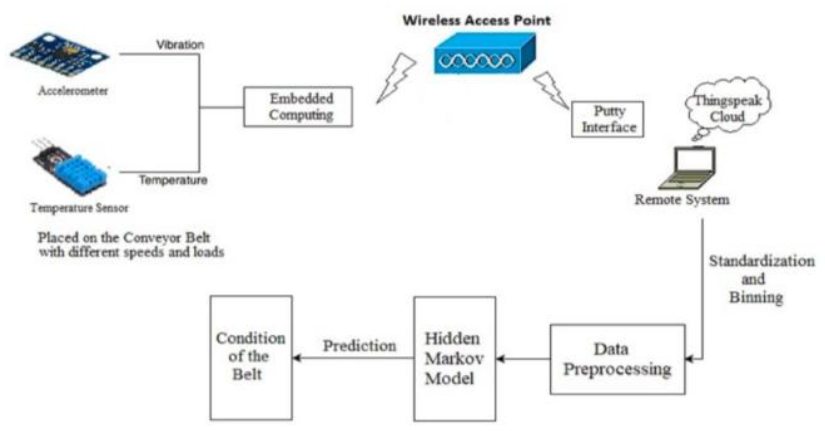

Figure 1: Proposed Model

The system consists of various sensors such as temperature, vibration, accelerometer, etc. Wireless sensor systems are used for the communication of sensor data wirelessly to the computer placed at a distance, to meet the industrial application. This type of wireless transmission of sensor data is provided to accommodate online monitoring of the state of machine, cloud storage of data and decision predictions can be applied with ease to the data which is acquired wirelessly. The designed embedded wireless system with temperature, acceleration sensors sense the equipment's data. The data acquired from these sensors is aggregated and is transferred to cloud via an access point setup. 
This data acquired is stored on cloud and is continuously monitored. Any deviation of the normal functioning of the equipment can be identified through the data. The data can be analysed to make optimal predictions.

The wireless sensor network with temperature and acceleration sensors are interfaced to the embedded board. Functioning of each sensor is listed below as an example:

- The temperature sensor is used to detect the ambient temperature. The equipment's temperature can be measured using this sensor. In case, if the conveyor belt is operated at a very high speed resulting in the overall temperature rise in the motor and belt, can be captured using this sensor. Also, if very hot substances are kept over the conveyor belt can be identified using this sensor.

- A 3-axis accelerometer with signal conditioned voltage outputs is the accelerometer sensor used. In tilt sensing applications, this sensor can measure the static acceleration of gravity as well the dynamic acceleration caused due to the motion of the equipment. This sensor provides data related to the $\mathrm{x}, \mathrm{y}$ and $\mathrm{z}$ axis values in the motion of the belt at a particular point where the sensor is placed.

\section{B. Data Acquisition and Data Cleaning}

The data from the sensors is collected from the Raspberry $\mathrm{Pi}$ and is sent to the ThingsSpeak cloud account, from which data could be downloaded to analyse it. ThingsSpeak provides a feature to view the live updating of data from the sensors, making it easier for the user to know if the Raspberry $\mathrm{Pi}$, along with sensors are placed properly on the conveyor belt. This collected data is downloaded in the form of csv file in our local machine so that it could be cleaned and analysed further. The data consists of 2 columns, the time at which the data is updated from Raspberry Pi to the cloud account and the value of the Accelerometer or temperature sensor. The Conveyor belt is run at different weights kept over it with different speeds.

\begin{tabular}{|c|c|c|}
\hline A & B & c \\
\hline & Hidden & Visible \\
\hline 0 & Speed5RPM_noweight & 551 \\
\hline 1 & Speed5RPM_noweight & 512 \\
\hline 2 & Speed5RPM_noweight & 543 \\
\hline 3 & Speed5RPM_noweight & 500 \\
\hline 4 & Speed5RPM_noweight & 532 \\
\hline
\end{tabular}

Figure 2: Glimpse of the dataset without data cleaning and which is in accordance with the Hidden Markov Chain

Data cleaning plays a major role in elimination of improper data points, which do not impact the predictive data model. The noisy data points were removed along with data points due to hardware malfunctions. Next, Data Standardization which is used to compare scores between different variables has been applied on the data points, which involves setting different variables on the same scale. To Standardize the variables, mean and standard deviation are calculated for each variable, following which the mean is subtracted from each variable and divided by the standard deviation. Then, Data Binning is used to create various bins to place the above standardized values into those bins so that we can arrive at a computation matrix consisting of all the probabilities. This technique is also known as Discretization, used in data pre-processing and statistics. The above data after data cleaning and pre-processing is used for analysing.

\begin{tabular}{|c|c|c|r|r|}
\hline A & \multicolumn{1}{|c|}{ B } & \multicolumn{1}{c|}{ E } \\
\hline Hidden & Visible_Continuous & Visible_Discrete_Standardized & Visible__iscrete_roundOff \\
\hline OSpeed5RPM_noweight & 551 & 0.343284608 & 2 \\
\hline 1 Speed5RPM_noweight & 512 & 0.649751287 & 4 \\
\hline 2 Speed5RPM_noweight & 543 & 0.139584937 & 1 \\
\hline 3 Speed5RPM_noweight & 500 & 0.955300793 & 5 \\
\hline 4 Speed5RPM_noweight & 532 & 0.14050211 & 1 \\
\hline
\end{tabular}

Figure 3: A glimpse of the dataset after applying the pre-processing techniques (Standardization and Binning)

\section{Mathematical Model for Analysis}

The model chosen for analysing the data is a Hidden Markov Model and basic probability rules to do the prediction of the data model. Hidden Markov Model (HMM) is statistic model where the system being modelled is assumed to be a Markov Model with some hidden states. It is an unsupervised Machine Learning Algorithm. However, Hidden Markov Model (HMM) is often trained using supervised learning method in case if the training data is available. HMM is a graphical model, which is generally used in predicting states (hidden) using sequential data like weather, text, speech etc. Markov Model has been used to model randomly changing systems such as weather patterns. In Markov Model all the states are visible or observable. In this project, the hidden state is the condition of the Conveyor Belt on which speed and weights it is running on and visible state is the vibration value of the accelerometer kept on the Conveyor Belt.

The most important point of Markov Model is that it states that the future state depends only on the current state and not on any other older states. This is known as Markov Property. Mathematically we can say, the probability of the state at time $t$ will only depend on time step $t-1$. In other words, the probability of $\mathrm{s}(\mathrm{t})$, if given $\mathrm{s}(\mathrm{t}-1)$, that is $\mathrm{p}(\mathrm{s}(\mathrm{t}) \mid \mathrm{s}(\mathrm{t}-1))$. This is known as First Order Markov Model. In case, the probability of the states at time $\mathrm{t}$ depends on time step $\mathrm{t}-1$ and $\mathrm{t}-2$, it's known as Second Order Markov Model. As there is an increase in the dependency of past events, the order increases. The 2nd Order Markov Model would be $\mathrm{p}(\mathrm{s}(\mathrm{t}) \mid \mathrm{s}(\mathrm{t}-1), \mathrm{s}(\mathrm{t}-2))$.

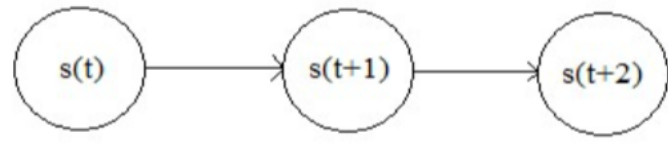

Figure 4: Second Order Markov Model

Basic Terminologies with respect to Markov Chain:

- Initial Probability Distribution: The System has to start from one state. That is the initial state of Markov Model (when time step $\mathrm{t}=0$ ) 


\section{Design and Development of Techniques for Equipment Health Monitoring System}

- Markov Chain: There are basic 4 types of Markov Models. When the system is fully observable and autonomous it's called Markov Chain. The Markov Chain consists of the following parameters: A set of $\mathrm{M}$ states, an initial probability, and a transition probability matrix A

- Final /Absorbing State: When the transition probabilities of any step to other steps are zero except for itself then it is known as a Final/Absorbing State. So when the system enters into the Final/Absorbing State, it never leaves.

- Hidden Markov Model: In Hidden Markov Model the state of the system will be hidden (unknown), however at every time step $\mathrm{t}$ the system in states(t) will emit an observable/visible symbol $v(t)$. An example of Hidden Markov Model in the below diagram.

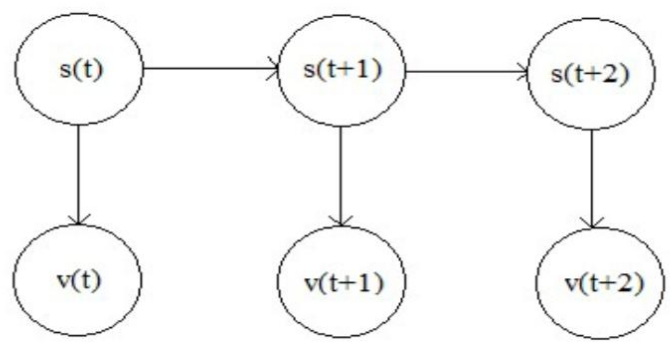

Figure 5: Hidden Markov Model

In this project, the probability matrix is created using python programming language which is of order, MXN, where $M$ is the number of conditions under which the Conveyor Belt is tested an $\mathrm{N}$ is the number of bins present in the dataset. Then the probability of each of the above point in the matrix is calculated for that particular condition in which the Conveyor Belt is running. The future data points can be a potential input to the conveyor belt, or in other words, the value of the accelerometer for the vibrations it is subjected to can be predicted if the Belt is running normally in a healthy environment. Using this technique we can actually monitor the health of the belt whether it is running within proper speed ranges or if the belt is not in proper working conditions.

The Hidden Markov Model is used in the prediction of the hidden state of the machine, that is the condition of the machine, given the visible state of the machine, that is, the accelerometer value or temperature sensor value. Using a probability matrix which is generated, HMM can predict the hidden state, that is the condition of the machine on which it is operated. Any variation from this predicted value of the hidden state, can be considered as faulty behaviour of the machine and human intervention is required.

\section{Algorithm}

1. Connect the sensors to embedded boards and place it on the equipment to be monitored. In our case, Conveyor belt deployed at Honeywell laboratory at Information Science and Engineering laboratory, M.S Ramaiah Institute of Technology.

2. Set the belt in motion at different speeds and collect values at various points with different loads/weights placed on the belt.
3. The collected values are directly stored on ThingsSpeak Cloud where data can be downloaded and visualized.

4. Data Cleaning is performed on the obtained dataset to remove null and missing values in the dataset, data standardization is applied to the data and further data is discretized.

5. The signature of the belt is obtained by plotting the dataset values using matplotlib library.

6. The forward and backward learning algorithm is applied to obtain a probability matrix in the following way:

- Forward Algorithm: To derive the probability of the next time step, in Forward Algorithm we use the computed probability on the current time step. Hence it is computationally more efficient $\mathrm{O}\left(\mathrm{N}^{\wedge} 2 . \mathrm{T}\right)$. If provided with a sequence of visible state $\mathrm{V}$, to make the algorithm recursive, the probability of the Hidden Markov Model being at a hidden state at a specific time step $\mathrm{t}$ needs to be determined.

- Backward Algorithm: This is the time reversal of the Forward Algorithm. Along with finding the probability that the machine will be in hidden state $\mathrm{S}$ at time $\mathrm{t}$, the backward Algorithm will also generate the remaining part of the sequence of the visible symbol V. Both these algorithms have been used in this paper.

7. Thus, the state in which the conveyor belt is running is displayed on the screen. Any abnormalities on the belt are also predicted by the obtained results.

8. Given any value of the conveyor belt, the above generated data model would be able to predict if the conveyor belt is running at expected conditions or if any human intervention is required.

\section{IMPLEMENTATION}

The wireless sensor network with temperature and accelerometer sensors are interfaced to an embedded board. In our case, Raspberry Pi2 board along with - temperature and accelerometer sensors are deployed on the Conveyor test rig, set up at Honeywell research lab, Dept of Information Science and Engineering, M.S Ramaiah Institute of Technology.

Functioning of each of these sensors are stated below:

1. Temperature Sensor: It is used to detect the temperature of the surrounding region. With respect to this project, it is used to determine the temperature at which the equipment is operating.

2. Accelerometer Sensor: The sensor used is a 3 -axis accelerometer with signal conditional voltage output. It is used to measure the tilt of the equipment is $3 \operatorname{axes}(\mathrm{x}, \mathrm{y}, \mathrm{z}$ axis). 
The sensor data is collected over the Conveyor Belt, with 3 such weights and 3 speed values and integration of these two factors. The belt has been run at speed of 5rpm (rotations per minute) to $15 \mathrm{rpm}$ with no weights, weight of $2 \mathrm{~kg}$ placed on it, weight of $4 \mathrm{~kg}$ placed on it. The dataset thus obtained consists of these above nine combinations. The data is collected by the Raspberry Pi and transmitted to a configured cloud account through which data can be downloaded and accessible. For each of the above condition, 100 data points have been collected from the Conveyor Belt.

The vibrations of the belt collected, when transferred to the ThingsSpeak account will have the GPS (exact location) on where exactly those vibrations on the belt are captured, thus enabling users to know exactly where the vibrations are recorded. This feature makes it very useful in cases of Conveyor Belt covering very large distance (oil refineries, coal mining etc) to know at which part of the belt the vibrations are recorded.

\section{RESULT}

The accelerometer sensor values with respect to time are shown in figure 4 for better visualization. The $x$-axis represents time and the $y$-axis represents the value gained by the accelerometer sensor. The ThingsSpeak cloud takes real time data from the sensors and displays on the local system alongside temperature. The data acquisition is done in the xlsx format and further analysis is done.

The prediction done is by using Hidden Markov Model with forward and backward algorithm. The algorithm is trained for several values from the accelerometer. The results are presented in the form of a matrix that are used to understand and eliminate fault data. Different signatures are gained and understood for detailed analysis.

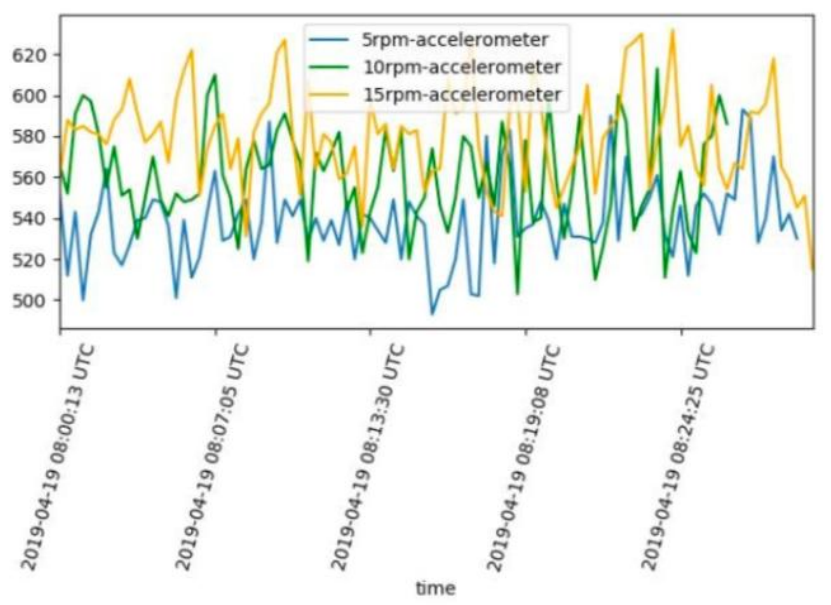

Figure 6: Accelerometer values with time

In our case, the values from the sensors obtained through the ThingsSpeak Cloud are processed. These processed values form the training dataset. When a new accelerometer test value is encountered, the proposed model predicts the condition of the equipment, for example, "Speed 5rpm no Weight" as shown in Figure 7, indicates that the Equipment is running at a speed of $5 \mathrm{rpm}$ and there is no load on the machine. In case of an unexpected accelerometer value, the user will be alerted with an Abnormality Message as shown in Figure 8.

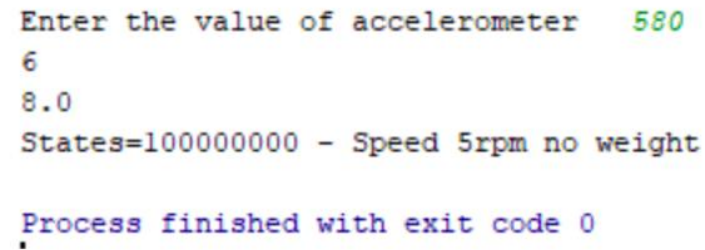

Figure 7: State of belt shown to the user based on values received from sensors.

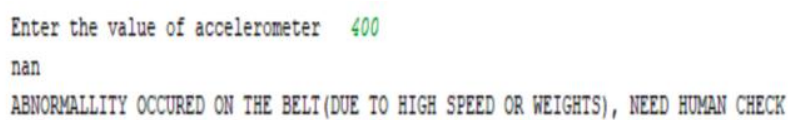

Figure 8: An alert shown to user in case of abnormalities.

\section{CONCLUSION}

This project offers a design of automated equipment health monitoring system that tracks the changes taking place in the equipment at regular intervals. The sensors are deployed to detect vibration parameters and speed of the conveyor belt. We have designed and developed a system where the sensors are tested for different cases and the results are verified and also, the prediction is carried out by Hidden Markovian Model. In most of the cases, the monitoring of the conveyor belt is done and the deployment of sensors were tested, periodic collection of data is verified.

This embedded wireless system with the sensors can be used to detect any change in the vibration of an equipment. Hidden Markovian Model is used for the case of prediction of different cases and signatures. The signatures repeat over a time period, making it easier for prediction of the entire system that is being monitored.

This model can be used for any vibrational or any mobile equipment such as boilers, drilling machines and other such tools. Hidden Markovian Model can be used for prediction of states of that equipment in any automated industry which will reduce the manpower and also, accuracy can be improved by using data collection periodically. During this setup and after some time period, automatic monitoring can be made regarding the equipment health of the industry.

\section{ACKNOWLEDGEMENT}

We thank the Department of Information Science and Engineering, for providing us to use the facilities of Honeywell Research Lab, and to use the test rig Conveyor Belt to carry out our experimentation and guidance by the faculty. We also thank the Principal of RIT, for his advice and timely support during our graduation course and project work. 


\section{Design and Development of Techniques for Equipment Health Monitoring System}

\section{REFERENCES}

1. Reni Suryanita, Azlan Adnan, "Application of Neural Networks in Bridge Health Prediction based on Acceleration and Displacement Data Domain" Proceedings of the International Multi Conference of Engineers and Computer Scientists, Vol I, 2013.

2. Kavita Kumari, Inderdeep Kaur Aulakh, Amol P Bhondekar, "Structural Health Monitoring System Using Wireless Sensor Network" Conference, April 2015.

3. M. Sader, T. Jeinsch, R. Noack, "A Model-Based Approach to Fault Detection and Abrasion Depending Maintenance on Belt Conveyor Systems" Germany.

4. David Rojas, John Barrett, "Structural Health Monitoring System Using Wireless Sensor Network" Conference, June 2017.

5. B. P Vijaya Kumar and P. Venkataraman, Prediction-based location Management using multilayer neural networks, J. Indian Inst Sci., 82,721 copyright Indian Institute of Science.

6. James Moyne, Jimmy Iskandar and Parris Hawkins, Toysha Walker ,Avi Furest, Bryan Pollard, David stark. Deploying an equipment Health Monitoring Dashboard and accessing Predictive maintenance, Advanced Semiconductor Manufacturing Conference(ASMC),2013, 24th Annual SEMI

7. Ainul Anam Shahjamal Khan, Adittya Ranjan Chowdhury, Md. Shahriar Haque, Monitoring and Detecting Health of a Single Phase Induction Motor Using Data Acquisition Interface (DAI) module with Artificial Neural Network, WSEAS Transactions On Systems And Control,2014, Vol 9, p229, 2014.

8. Marc Roelands, Bell Labs, Alcatel-Lucent, Antwerp, Belgium, IOT Service Platform Enhancement Through 'In- Situ' Machine Learning Of Real-World Knowledge, Local Computers Network Workshops(LCN), IEEE 38thConference, 2013

\section{AUTHORS PROFILE}

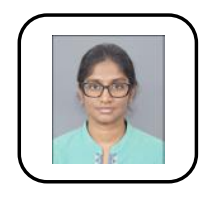

Vasireddy Prabha Kiranmai received her Bachelor's degree in Information Science and Engineering from Ramaiah Institute of Technology, Bengaluru. She was awarded a medal for her outstanding academic performance by the Institute. She also involves herself in reading Research papers and keeps herself updated on latest innovations. She has contributed to a chapter, "Security and Privacy Issues in IoT: A Platform for Fog Computing" in the IGI Global Journal, "The Rise of Fog Computing in the Digital Era". She has been certified an Elite grade by Indian Institute of Technology, Madras for Machine Learning course conducted by NPTEL in 2018. She has actively participated in the Big Data workshop conducted by Indian Institute of Science, Pravega. She has good foundation in computer science and mathematics, and would like to enhance her knowledge in cross-domain aspects. She is actively involved in learning about the areas which involve intersection of two or more disciples of computer science. She takes interest in areas like Machine Learning, Cloud Technologies and Cyber Security. She is immensely passionate about building products using these technologies to reduce human efforts.

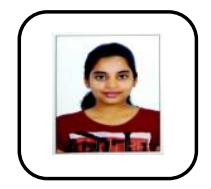

Sharmitha S Bysani. She received the Bachelor of Engineering degree as a Gold Medalist in Information Science and Engineering from Ramaiah Institute of Technology, Bangalore. Her research interests include Internet of Things, Machine Learning and Ethical Hacking. She is enthusiastic about exploring new technologies and revising her skills to adapt to the changing technological environments around the world. Taking up and being a part of innovations concerning the upliftment of the society are her top priorities. She has also worked with hands-on exposure in domains like Machine Learning, Image Processing and IoT. She has also published a Book Chapter on "Security and Privacy Issues in IoT: A Platform for Fog Computing".

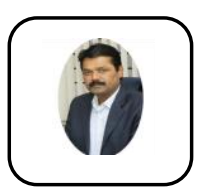

Vijay Kumar B P [SMIEEE, Fellow IETE, LMISTE, SMIACSIT] Ph.D in Electrical Communication Engineering (2003), Indian Institute of Science, Bangalore, M. Tech in Computer Science and Technology, India Institute of Technology) (1993), BE in Electronics and Communication, First class with distinction, Mysore University (1987),. Presently he is a Professor and Head, Information Science and Engineering., Dept, M S Ramaiah Institute of Tech., Bangalore. His research interest Applying Computational Intelligence - to meet the future smart-network's requirement such as cognitive computing approaches for intelligent networks. Involved in designing an adaptable, reliable, scalable and stabilizable routing algorithms for mobile Adhoc and sensor networks, and also includes mobility based issues and context-aware service strategies by using intelligent techniques, i.e., swarm \& bio-inspired computing and Neural Networks to solve the optimization or/and function approx. problems in mobile networks for better services. He is having 26 years of Teaching and 20 years of Research experience. He has published around 70 papers which include journals, conferences and project proposals. His name is listed in Marquis Who's Who in the World, and Marquis Who's Who in Asia, 2012,14.

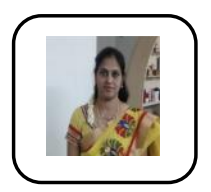

Ms. Kusuma S. M. [MIEEE, LMISTE] is working as an Assistant Professor in Telecommunication Engineering department, RIT. Completed her undergraduate from Mysore University in Electronics and Communication Engineering., M. Tech in VLSI and Embedded systems from Visvesvaraya Technological University, and presently pursuing her research work towards Sensors deployment in Embedded Sensor networks. Her areas of interests are Wireless Sensor Networks, Wireless Mobile Communication, Internet of Things, Embedded System Design, MIMO Technology, Machine learning and 4g/5g Technology. Involved in community outreach through project based learning for undergraduate engineering students. She has published around 10 papers which includes journals, conferences and project proposals in the said areas. 